\title{
THE IMPORTANCE OF GAS SCATTERING PROCESSES ON THE STOICHIOMETRY DEVIATIONS OF LASER DEPOSITED FILMS
}

J.Gonzalo, C.N.Afonso

Instituto de Optica, CSIC, Serrano 121, 28006 Madrid, Spain

J.Perrière, R.Gómez San Roman

Groupe de Physique des Solides, Université Paris VII et VI, URA 17, Tour 23, 2 Place Jussieu, 75251 Paris Cedex 05, France

\section{ABSTRACT:}

Pulsed laser deposition is a widely used technique to produce high quality films of complex oxides because of its stoichiometric character. Nevertheless, it is well known that the presence of a gas environment during growth which is usually required to produce good quality films, is also known to lead to some stoichiometry deviations. Several mechanisms like preferential evaporation or re-sputtering from the growing film, reactions in the gas phase and scattering by the foreign gas prior to deposition have been reported to account for these deviations. The aim of this work is to show that gas scattering processes are the only one responsible of these deviations.

Ablation of a $\mathrm{BiSrCaCuO}$ (2212) target is performed with an $\mathrm{ArF}$ excimer laser $\left(\lambda=193 \mathrm{~nm}, \tau=12 \mathrm{~ns}\right.$ FWHM) in a vacuum chamber evacuated to $4 \times 10^{-8}$ mbar. The films are grown in vacuum and in a reactive $\left(\mathrm{O}_{2}\right)$ and inert $(\mathrm{Ar})$ atmospheres. Two Si substrates are properly located in the chamber in order to collect material from the scattering of the ablated species by the foreign atmosphere, from the re-emission processes from the growing films or from both processes. The composition of the films is determined by ion beam techniques (Rutherford Backscattering Spectrometry and Nuclear Reaction Analysis).

The results show that evaporation or re-sputtering processes from the growing film are very weak and may affect to $1 \%$ of the deposited material at the most. Whereas films deposited in vacuum are nearly stoichiometric, films grown in the presence of a foreign gas present significant deviations, the deviations being qualitatively similar in Ar and oxygen environments. The results show clearly that these deviations are related to scattering processes prior to deposition and not to chemical reactions in the gas phase or re-emission processes from the growing film. These scattering processes are more important for the lighter species, thus explaining the Bi enrichment of the films. 